\title{
Clinical Features, Strain Distribution, Antifungal Resistance and Prognosis of Patients with Non-albicans Candidemia: A Retrospective Observational Study
}

Fengqi Liu, ${ }^{1,2, *}$ Li Zhong, ${ }^{1,2, *}$ Feifei Zhou, ${ }^{3, *}$ Cheng Zheng, ${ }^{1,4}$ Kai Zhang,' Jiachang Cai, ${ }^{5}$ Hongwei Zhou, (iD $)^{5}$ Kankai Tang, ${ }^{2}$ Zhaohui Dong, ${ }^{2}$ Wei Cui,' Gensheng Zhang (D')

'Department of Critical Care Medicine, Second Affiliated Hospital, Zhejiang University School of Medicine, Hangzhou, Zhejiang, 310009, People's Republic of China; ${ }^{2}$ Department of Critical Care Medicine, First Affiliated Hospital, Huzhou Teachers College, The First People's Hospital of Huzhou, Huzhou, 3I3000, Zhejiang, People's Republic of China; ${ }^{3}$ Department of Critical Care Medicine, Ningbo Medical Center, Li Huili Hospital, Ningbo, Zhejiang, 315040 , People's Republic of China; ${ }^{4}$ Department of Critical Care Medicine, Taizhou Municipal Hospital, Taizhou, Zhejiang, 318000, People's Republic of China; ${ }^{5}$ Department of Clinical Microbiology Laboratory, Second Affiliated Hospital, Zhejiang University School of Medicine, Hangzhou, 310009, People's Republic of China

*These authors contributed equally to this work

Correspondence: Gensheng Zhang; Wei Cui

Department of Critical Care Medicine, Second Affiliated Hospital, Zhejiang University School of Medicine, cc88 Jiefang Road, Hangzhou, 310009, People's Republic of China

Tel +86-57I-8778-3636;

$+86-571-8778-355$ I

Fax +86-57I- 87022776

Email genshengzhang@zju.edu.cn;

zricu@zju.edu.cn
Purpose: Candida albicans ( $C$. albicans) candidemia has been well reported in previous studies, while research on non-albicans Candida (NAC) bloodstream infections remains poorly explored. Therefore, the present study aimed to investigate the clinical characteristics and outcomes of patients with NAC candidemia.

Patients and Methods: We recruited inpatients with candidemia from January 2013 to June 2020 in a tertiary hospital for this retrospective observational study.

Results: A total of 301 patients with candidemia were recruited in the current study, including $161(53.5 \%)$ patients with NAC candidemia. The main pathogens in NAC candidemia were Candida tropicalis (C. tropicalis) (23.9\%), Candida parapsilosis $(15.6 \%)$ and Candida glabrata (10.3\%). Patients with NAC candidemia had more medical admissions $(P=0.034)$, a higher percentage of hematological malignancies $(P=0.007)$, a higher frequency of antifungal exposure ( $P=0.012)$, and more indwelling peripherally inserted central catheters $(P=0.002)$ than those with $C$. albicans candidemia. In a multivariable analysis, prior antifungal exposure was independently related to NAC candidemia (adjusted odds ratio [aOR], 0.312; 95\% confidence interval [CI], 0.113-0.859). Additionally, NAC was obviously resistant to azoles, especially $C$. tropicalis had a high cross-resistance to azoles. However, no significant differences were noted in the mortality rates at 14 days, 28 days and 60 days between these two groups.

Conclusion: NAC is dominant in candidemia, and prior antifungal exposure is an independent risk factor. Of note, although the outcomes of NAC and C. albicans candidemia are similar, drug resistance to specific azoles as well as cross-resistance frequently occurs in patients with NAC candidemia, and this drug resistance deserves attention in clinical practice and further in-depth investigation.

Keywords: non-albicans candidemia, clinical features, risk factor, cross-resistance

\section{Introduction}

With the wide usage of antibiotics, immunosuppressive agents and glucocorticoids, candidemia has become common as a bloodstream infection (BSI). It often occurs in patients receiving complex surgery, organ transplantation, intravascular catheters, and total parenteral nutrition (TPN), and in patients who have hematologic malignancies or who are in the intensive care unit (ICU). ${ }^{1,2}$ The prevalence of candidemia varies in different regions, ${ }^{1,3,4}$ ranging from a relatively low occurrence of $0.32 / 1000$ admissions 
in Southwest China to a high incidence of 2.49/1000 admissions in Brazil. ${ }^{3,4}$ Although a rapid diagnosis and timely treatment have been developed, the mortality of invasive candidemia is still relatively high, ranging from $22 \%$ to $75 \%$. ${ }^{2,5,6}$

The most common pathogen causing candidemia is Candida albicans (C. albicans), but epidemiological investigations in recent years have shown that the incidence of non-albicans Candida (NAC) in candidemia is increasing year by year and is mainly composed of Candida tropicalis (C. tropicalis), Candida parapsilosis (C. parapsilosis) and Candida glabrata (C. glabrata). ${ }^{5}$ In addition, $C$. albicans is highly sensitive to commonly used clinically antifungal drugs, while the drug resistance seen in NAC is steadily increasing. ${ }^{7,8}$ Especially, two NAC species (spp.), C. glabrata and C. tropicalis, have demonstrated a higher drug resistance to azoles than other Candida spp. ${ }^{7}$ Patients with NAC candidemia generally are more likely to have neutropenia and are more likely to have received TPN, glucocorticoids and central venous catheters (CVCs), whereas patients with C. albicans candidemia are more likely to have indwelling urethral catheters, are more likely to have candiduria and are more likely to be admitted to the ICU. ${ }^{9-11}$

In previous studies, some differences have been reported between C. albicans and NAC candidemia with regard to the clinical characteristics and prognostic factors. ${ }^{7-11}$ However, several limitations are shown as follows: (1) One study identified that the presence of a urethral catheter was an independent risk factor for $C$. albicans candidemia, ${ }^{10}$ and glucocorticosteroids and $\mathrm{CVC}$ were independent risk factors for NAC candidemia in another study. ${ }^{11}$ However, whether these clinical features are significantly different between these two groups remains unclear. (2) A previous study found that patients with $C$. albicans candidemia had a higher rate of ICU hospitalization, but there was no significant difference in the hospital mortality; ${ }^{9}$ This result was completely contrary to another study (there was no difference in the ICU hospitalization rate, but there was a higher mortality rate for NAC candidemia). ${ }^{10}$ Therefore, whether the clinical outcomes of NAC candidemia are better or worse than those of $C$. albicans candidemia remains unclear. (3) Although the distribution and antifungal resistance of Candida spp. have been well reported in a multicenter large-scale study by China CHIF-NET, more information about the demographic and clinical characteristics is still lacking, and this information is needed to draw valid conclusions. ${ }^{8}$
Based on the previous results and controversies described above, we hypothesized that patients with NAC candidemia might have some specific risk factors, a more severe situation of drug resistence like azole and a worse prognosis than those with $C$. albicans candidemia. To address this hypothesis, we attempted to analyze the clinical features, strain distribution, antifungal resistance and prognosis of NAC candidemia compared with C. albicans candidemia in the current study.

\section{Patients and Methods}

\section{Study Design and Patients}

The present single-center retrospective study was carried out in a tertiary medical teaching hospital, the Second Affiliated Hospital, Zhejiang University School of Medicine, China. The Ethics Committee of the Second Affiliated Hospital, Zhejiang University School of Medicine approved this study protocol (No. 2020-744). Due to the retrospective analysis, the Ethics Committee decided to waive the need for informed consent of patients.

The results of 476 positive blood culture samples from the microbial laboratory between January 2013 and June 2020 were initially analyzed (Figure 1). Among them, there were 123 duplicated Candida specimens, and any duplicate specimens from the same patient were excluded. Then, we excluded the following patients: 1) age $<18$ years old; 2) Candida was considered as nonpathogenic; and 3) the case data were incomplete or missing. Consequently, 52 patients were excluded, including one patient less than 18 years old, 34 patients with nonpathogenic Candida and 17 patients with incomplete or missing data. Finally, 301 patients with candidemia were recruited, with 140 cases and 161 cases of C. albicans candidemia and NAC candidemia, respectively.

\section{Study Variables}

The following patients' medical variables were retrieved from the electronic medical record system, and the variables included basic information such as age, sex, previous medical history, and several assessments [eg, Charlson Comorbidity Index (CCI) score, acute physiology and chronic health evaluation (APACHE) II score and sequential organ failure assessment (SOFA) score within 24 hours after Candida BSI]. Other information, including a history of invasive procedures, previous exposure, previous treatment (eg, chemotherapy drugs, radiotherapy, immunosuppressive agents, surgery, mechanical ventilation), laboratory examinations 


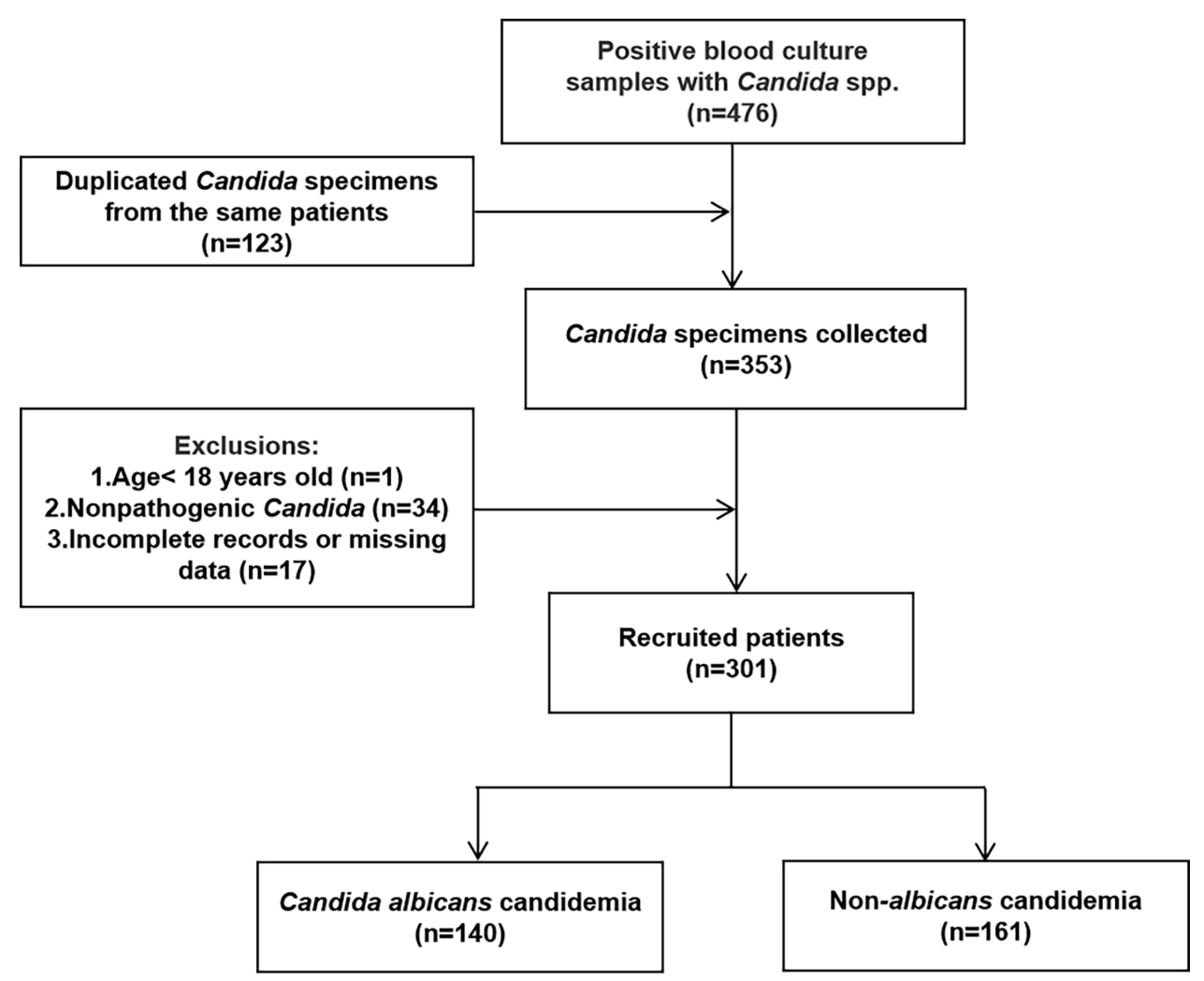

Figure I Flow diagram of patient recruitment.

(eg, blood cells, liver function, and kidney function), and the microbiological data (Candida spp., concomitant bacterial infection or not, antifungal susceptibilities and crossresistance to azoles in vitro), were also documented. In addition, the main treatments for candidemia, such as fluid resuscitation, vasoactive drugs, renal replacement therapy (RRT) and antifungal drugs, and the outcomes, such as mortality rates at 14 days, 28 days and 60 days, were collected.

\section{Species Identification and Microbiological Assays}

Candida spp. identification and drug susceptibility testing were conducted as described in our previous study. ${ }^{12}$ In brief, blood cultures were drawn under aseptic conditions, and then matrix-assisted laser desorption/ionization time-of -flight mass spectrometry (MALDI-TOF MS) (Bruker Daltonik GmbH, Bremen, Germany) was used to identify Candida spp. After species confirmation, all of the Candida isolates were subjected to antifungal susceptibility tests using the ATB FUNGUS 3 panel of bioMerieux company in France. Experimental assessments of drug susceptibility for Candida were based on the clinical breakpoints specified by the Clinical Laboratory Standards Institute. ${ }^{13,14}$

\section{Study Definitions}

Candidemia was diagnosed when there was an isolate of at least one species of Candida from the blood cultures accompanied by signs and symptoms of infection. Nonpathogenic candidemia isolates were considered contaminants and were defined as a single positive blood culture of Candida without the clinical manifestations. ${ }^{11}$ The definition of catheterrelated candidemia was on the basis of the Infectious Diseases Society of America and was defined as follows: 1) the isolates from a catheter tip culture was proven to be identical to the isolates in least one percutaneous peripheral blood culture; or 2) the transcatheter and peripheral blood samples cultured the same Candida spp., and met the catheter-related bloodstream infection (CRBSI) criteria. ${ }^{15}$ The diagnostic criteria for septic shock was based on the definition by Sepsis-3. ${ }^{16}$ When a blood bacterial culture was positive before or within 48 hours after the onset of candidemia, it was considered a concomitant bacteremia, ${ }^{4}$ except for the common skin microbiota (eg, Corynebacterium spp., Streptococci, Bacillus spp., Coagulase-negative staphylococci and Lactobacillus spp.), which are possible contaminants. Unless two or more consecutive venipuncture samples cultured the above microorganisms, these isolates were 
considered pathogens. ${ }^{12,17}$ The antifungal treatment was considered adequate if: (1) the antifungal agent was administered empirically within the first 48 hours of positive culture; (2) the Candida isolates were sensitive to the selected antifungal drugs on a sensitivity test; and (3) the dosage of antifungal drugs was selected according to the clinical guidelines recommended by the Infectious Diseases Society of America. ${ }^{18,19}$

\section{Statistical Analysis}

All statistical analyses were performed to identify the risk factors for NAC candidemia in comparison with C. albicans candidemia by using the statistical package SPSS 23.0 (IBM Corp, Armonk, NY, USA), and a $P<0.05$ was considered statistically significant. First, all quantitative data were first tested for normality. If the test results conformed to a normal distribution, the mean \pm standard deviation was used to represent the continuous variables; otherwise the median and interquartile range (IQR) were used instead. Then, Student's $t$-test or the Mann-Whitney U was used for comparison. All enumeration data were represented as $\mathrm{N}(\%)$, and the chi-square test was used for comparisons between the two groups. In the univariate analysis, the variables with a significant $P<0.05$ level were considered candidate variables for establishing a stepwise logistic regression multivariate model, which was used to identify the independent risk factors associated with NAC candidemia. The 28-day survival curves of $C$. albicans and NAC candidemia were depicted by a Kaplan-Meier survival analysis, and the difference was evaluated by the Log rank test.

\section{Results}

\section{Patient Characteristics}

Table 1 outlines the baseline characteristics of the recruited patients. The median age of these patients was 66 years (IQR, $53.0-75.5)$, and $64.1 \%(193 / 301)$ were male. A total of $66.4 \%$ (200/301) of all patients with candidemia occurred at an age of older than 60 years old. The proportion of patients over 60 years of age with NAC candidemia was lower than that with C. albicans candidemia $(60.9 \%$ vs $72.9 \%, P<0.05)$. The majority of patients with candidemia were from the ICU (64.5\%), followed by surgical wards $(20.9 \%)$ and medical wards (14.6\%), and $91.0 \%(274 / 301)$ of these candidemia cases were nosocomial infections. In terms of comorbidities, gastrointestinal (GI) disease (31.9\%), solid tumors (23.6\%), and diabetes mellitus (18.3\%) were common complications. A lower proportion of diabetes mellitus $(13.0 \%$ vs $24.5 \%$,
$P<0.05)$ and GI diseases $(26.1 \%$ vs $38.6 \%, P<0.05)$ were observed in patients with NAC candidemia, but more hematological malignancies $(6.8 \%$ vs $0.7 \%, P<0.05)$ were observed in patients with NAC candidemia than in patients with C. albicans candidemia. There were no statistically significant differences between the two groups in terms of the CCI score, APACHE II score or SOFA score among all the patients (all $P>0.05$ ) (Table 1). The percentage of antibiotic exposure before the onset of candidemia was $86.0 \%$, followed by TPN and surgery for more than $50 \%$ of the patients. Compared to C. albicans candidemia, patients with NAC candidemia had a lower rate of surgery $(47.2 \%$ vs $67.9 \%, P<0.001)$, especially abdominal surgery $(14.3 \%$ vs $34.3 \%, P<0.001)$. This result was consistent with the fact that most $C$. albicans candidemia cases were from surgical wards ( $26.4 \%$ vs $16.1 \%, P<0.05)$. In contrast, patients with NAC candidemia were more likely to be exposed to antifungal drugs $(12,4 \%$ vs $4.3 \%, P<0.05)$. In addition, more than $70 \%$ of patients with candidemia had invasive procedures such as CVCs, urinary catheters, and gastric catheters. Compared with catheterization of patients with $C$. albicans candidemia, indwelling arterial catheters and CVCs were less common in patients with NAC candidemia $(26.7 \%$ vs $39.3 \%$ and $67.1 \%$ vs $84.3 \%$, respectively, both $P<0.05)$, as were indwelling abdominal drainage tubes (13.7\% vs $32.1 \%, P<0.001)$. However, the presence of a peripherally inserted central catheter (PICC) was more frequent in patients with NAC candidemia $(24.8 \%$ vs $10.7 \%, P<0.05)$.

\section{Biological Parameters}

In terms of biological parameters, patients with NAC candidemia were more likely to have a white blood cell (WBC) count less than $4 \times 10^{9} / \mathrm{L} \quad(16.8 \%$ vs $5.0 \%$, $P=0.001)$, a lower neutrophil count (NC) (median $\times 10^{9} /$ $\mathrm{L}, 7.0$ vs 8.6), a lower neutrophil to lymphocyte ratio (NLR) (median, 9.2 vs 12.3), and a lower total bilirubin (TB) (median $\mu \mathrm{mol} / \mathrm{L}, 15.0$ vs 18.5 ) (all $P<0.05$ ) compared to patients with C. albicans candidemia (Table 2).

\section{Independent Risk Factors for NAC Candidemia}

Several variables with significant $p$ values in the univariate analysis are described in Table3. After the multivariate regression model analysis of these variables, it was noted that prior antifungal exposure was independently associated with an increased risk of NAC candidemia (adjusted odds ratio [aOR], 0.312; 95\% confidence interval [CI], 0.113-0.859). Patients with diabetes mellitus had 
Table I Baseline Characteristics of Patients with C. albicans and NAC Candidemia

\begin{tabular}{|c|c|c|c|c|}
\hline Characteristics & Total $(n=30 I)$ & C. albicans $(n=140)$ & $\operatorname{NAC}(n=161)$ & $P$-value \\
\hline Age, median years (IQR) & $66.0(53.0,75.5)$ & $68.0(58.2,75.0)$ & $64.0(49.0,77.5)$ & 0.338 \\
\hline Age ( $\geq 60$ years), $n(\%)$ & $200(66.4 \%)$ & $102(72.9 \%)$ & $98(60.9 \%)$ & $0.028^{*}$ \\
\hline Male sex, $n(\%)$ & $193(64.1 \%)$ & $82(58.6 \%)$ & III(68.9\%) & 0.061 \\
\hline \multicolumn{5}{|l|}{ Ward } \\
\hline Medical ward, n(\%) & $44(14.6 \%)$ & $14(10.0 \%)$ & $30(18.6 \%)$ & $0.034 *$ \\
\hline Surgical ward, n(\%) & $63(20.9 \%)$ & $37(26.4 \%)$ & $26(16.1 \%)$ & $0.029 *$ \\
\hline ICU, n(\%) & $194(64.5 \%)$ & $89(63.6 \%)$ & $105(65.2 \%)$ & 0.766 \\
\hline Nosocomial infection, $\mathrm{n}(\%)$ & $274(91.0 \%)$ & $130(92.9 \%)$ & | $44(89.4 \%)$ & 0.301 \\
\hline \multicolumn{5}{|l|}{ Baseline comorbidities } \\
\hline Chronic pulmonary disease, $\mathrm{n}(\%)$ & $18(6.0 \%)$ & $8(5.7 \%)$ & $10(6.2 \%)$ & 0.856 \\
\hline Haematological malignancy, n(\%) & $12(4.0 \%)$ & $\mathrm{I}(0.7 \%)$ & II(6.8\%) & $0.007 *$ \\
\hline Chronic cardiac insufficiency, $n(\%)$ & $49(16.3 \%)$ & $29(20.7 \%)$ & $20(12.4 \%)$ & 0.052 \\
\hline Neurological disease, $n(\%)$ & $5 \mathrm{I}(16.9 \%)$ & $18(12.9 \%)$ & $33(20.5 \%)$ & 0.078 \\
\hline Diabetes mellitus, $\mathrm{n}(\%)$ & $55(18.3 \%)$ & $34(24.5 \%)$ & $21(13.0 \%)$ & $0.012^{*}$ \\
\hline Solid tumor, $n(\%)$ & $71(23.6 \%)$ & $40(28.6 \%)$ & $31(19.3 \%)$ & 0.058 \\
\hline Solid organ transplant recipient, $n(\%)$ & $5(1.7 \%)$ & $4(2.9 \%)$ & $I(0.6 \%)$ & 0.288 \\
\hline Chronic kidney disease, $\mathrm{n}(\%)$ & $28(9.3 \%)$ & $\mathrm{II}(7.9 \%)$ & $17(10.6 \%)$ & 0.421 \\
\hline Chronic liver disease, $n(\%)$ & $30(10.0 \%)$ & $17(12.1 \%)$ & $13(8.1 \%)$ & 0.240 \\
\hline Gastrointestinal disease, $n(\%)$ & $96(31.9 \%)$ & $54(38.6 \%)$ & $42(26.1 \%)$ & $0.020^{*}$ \\
\hline Severe burn, $n(\%)$ & $15(5.0 \%)$ & $4(2.9 \%)$ & II (6.8\%) & 0.114 \\
\hline $\mathrm{CCl}$, median (IQR) & $4.0(3.0,6.0)$ & $5.0(3.0,7.0)$ & $4.0(3.0,6.0)$ & 0.119 \\
\hline APACHE II score, median (IQR) & $17.0(12.0,22.5)$ & $\mid 7.0(\mid 1.2,23.7)$ & $16.0(12.0,22.0)$ & 0.711 \\
\hline SOFA score, median (IQR) & $6.0(3.0,9.0)$ & $6.0(3.0,9.0)$ & $6.00(3.0,9.5)$ & 0.670 \\
\hline \multicolumn{5}{|l|}{ Risk factors } \\
\hline Current and former smoker, $n(\%)$ & $99(32.9 \%)$ & $4 \mathrm{I}(29.3 \%)$ & $58(36.0 \%)$ & 0.215 \\
\hline Septic shock on admission, $n(\%)$ & $32(10.6 \%)$ & $20(14.3 \%)$ & $12(7.5 \%)$ & 0.055 \\
\hline Surgery, $n(\%)$ & $|7|(56.8 \%)$ & $95(67.9 \%)$ & $76(47.2 \%)$ & $0.000 * *$ \\
\hline Abdominal surgery, $n(\%)$ & $71(23.6 \%)$ & $48(34.3 \%)$ & $23(14.3 \%)$ & $0.000 * *$ \\
\hline Steroid therapy, $n(\%)$ & $15(5.0 \%)$ & $9(6.4 \%)$ & $6(3.7 \%)$ & 0.283 \\
\hline Immunosuppressive therapy, $\mathrm{n}(\%)$ & $8(2.7 \%)$ & $6(4.3 \%)$ & $2(1.2 \%)$ & 0.201 \\
\hline Chemotherapy/radiation, $\mathrm{n}(\%)$ & $23(7.6 \%)$ & $7(5.0 \%)$ & $16(9.9 \%)$ & 0.108 \\
\hline Blood transfusion, $n(\%)$ & $115(38.2 \%)$ & $60(42.9 \%)$ & $55(34.2 \%)$ & 0.121 \\
\hline Prior antifungal exposure ${ }^{a}, n(\%)$ & $26(8.6 \%)$ & $6(4.3 \%)$ & $20(12.4 \%)$ & $0.012^{*}$ \\
\hline Prior antibiotics exposure ${ }^{a}, n(\%)$ & $259(86.0 \%)$ & $12 \mid(86.4 \%)$ & |38(85.7\%) & 0.858 \\
\hline TPN, n(\%) & $198(65.8 \%)$ & $97(69.3 \%)$ & $101(62.7 \%)$ & 0.232 \\
\hline Neutropenia, $n(\%)$ & $17(5.6 \%)$ & $5(3.6 \%)$ & $12(7.5 \%)$ & 0.146 \\
\hline \multicolumn{5}{|l|}{ Invasive devices } \\
\hline Mechanical ventilation, $\mathrm{n}(\%)$ & $|7|(56.8 \%)$ & $77(55.0 \%)$ & $94(58.4 \%)$ & 0.554 \\
\hline Presence of CVC, $n(\%)$ & $226(75.1 \%)$ & $118(84.3 \%)$ & $108(67.1 \%)$ & $0.001 *$ \\
\hline Presence of PICC, $n(\%)$ & $55(18.3 \%)$ & $15(10.7 \%)$ & $40(24.8 \%)$ & $0.002 *$ \\
\hline Presence of arterial catheter, $n(\%)$ & $98(32.6 \%)$ & $55(39.3 \%)$ & $43(26.7 \%)$ & $0.020^{*}$ \\
\hline Presence of urethral catheter, $n(\%)$ & $267(88.7 \%)$ & $129(92.1 \%)$ & 138(85.7\%) & 0.079 \\
\hline Presence of gastric tube, $n(\%)$ & $246(77.4 \%)$ & $110(80.9 \%)$ & $119(73.9 \%)$ & 0.137 \\
\hline Presence of abdominal drainage tube, $n(\%)$ & $67(22.3 \%)$ & $45(32.1 \%)$ & $22(13.7 \%)$ & $0.000 * *$ \\
\hline Blood purification, $\mathrm{n}(\%)$ & $79(26.2 \%)$ & $38(27.1 \%)$ & $4 I(25.5 \%)$ & 0.724 \\
\hline
\end{tabular}


Table I (Continued).

\begin{tabular}{|l|l|l|l|l|}
\hline Characteristics & Total $(\mathbf{n = 3 0 I )}$ & C. albicans $(\mathbf{n = 1 4 0 )}$ & NAC $(\mathbf{n = 1 6 I )}$ & $\boldsymbol{P}$-value \\
\hline Prior hospital stay, median days (IQR) & $15.0(6.0,31.0)$ & $14.5(5.2,31.7)$ & $16.0(6.0,31.0)$ & 0.654 \\
\hline Prior ICU stay, median days (IQR) & $5.0(0.0,19.5)$ & $5.0(0.0,15.5)$ & $6.0(0.0,21.0)$ & 0.213 \\
\hline
\end{tabular}

Notes: $* P<0.05, * * P<0.001$. ${ }^{a}$ All patients received systemic drug therapy for $\geq 3$ days within 2 weeks before onset of candidemia.

Abbreviations: C. albicans, Candida albicans; NAC, non-albicans Candida; IQR, interquartile range; ICU, intensive care unit; CCI, Charlson Comorbidity Index; APACHE, acute physiology and chronic health evaluation; SOFA, sequential organ failure assessment; TPN, total parenteral nutrition; CVC, central venous catheter; PICC, peripherally inserted central catheter.

Table 2 Biological Parameters of Patients with C. albicans or NAC Candidemia

\begin{tabular}{|c|c|c|c|c|}
\hline Variables & Total $(n=301)$ & C. albicans $(n=\mid 40)$ & NAC $(n=161)$ & $P$-value \\
\hline Temperature $>38^{\circ} \mathrm{C}, \mathrm{n}(\%)$ & $222(73.8 \%)$ & $97(69.3 \%)$ & $125(77.6 \%)$ & 0.100 \\
\hline Temperature $<36^{\circ} \mathrm{C}, \mathrm{n}(\%)$ & $9(3.0 \%)$ & $3(2.1 \%)$ & $6(3.7 \%)$ & 0.642 \\
\hline \multicolumn{5}{|l|}{ Laboratory data } \\
\hline \multicolumn{5}{|l|}{$\mathrm{WBC}\left(\times 10^{9} / \mathrm{L}\right), \mathrm{n}(\%)$} \\
\hline$<4$ & $34(I 1.3 \%)$ & $7(5.0 \%)$ & $27(16.8 \%)$ & $0.001 *$ \\
\hline$>10$ & $127(42.2 \%)$ & $65(46.4 \%)$ & $62(38.5 \%)$ & 0.165 \\
\hline $\mathrm{NC}\left(\times 10^{9} / \mathrm{L}\right), \operatorname{median}(\mathrm{IQR})$ & $7.8(4.8,11.9)$ & $8.6(5.4,17.8)$ & $7.0(4.0,10.7)$ & $0.005^{*}$ \\
\hline LC(×109/L), median(IQR) & $0.7(0.3, I . I)$ & $0.7(0.4,1.0)$ & $0.7(0.3, I . I)$ & 0.585 \\
\hline NLR, median(IQR) & II.4(6.3,19.9) & I2.3(8.2,23.0) & $9.2(5.5,17.5)$ & $0.003 *$ \\
\hline Anaemia, n(\%) & $267(88.7 \%)$ & $124(88.6 \%)$ & $143(88.8 \%)$ & 0.946 \\
\hline Thrombocytopaenia, n(\%) & $152(50.5 \%)$ & $71(50.7 \%)$ & $81(50.3 \%)$ & 0.944 \\
\hline Hypoproteinemia, n(\%) & $127(42.2 \%)$ & $56(40.0 \%)$ & $71(44.1 \%)$ & 0.473 \\
\hline $\mathrm{TB}(\mu \mathrm{mol} / \mathrm{L}), \operatorname{median}(\mathrm{IQR})$ & $16.0(\mid 1.0,31.0)$ & $18.5(|1| 0,35.6)$ & $15.0(10.0,28.0)$ & $0.029 *$ \\
\hline AST(U/L), median(IQR) & $39.0(26.0,65.5)$ & $43.0(27.0,75.5)$ & $36.0(25.0,57.5)$ & 0.080 \\
\hline $\operatorname{ALT}(\mathrm{U} / \mathrm{L})$, median(IQR) & $32.0(21.0,64.0)$ & $33 \cdot 5(21.0,64.8)$ & $30.0(19.5,63.0)$ & 0.356 \\
\hline Renal failure, $n(\%)$ & $64(21.3 \%)$ & $36(25.7 \%)$ & $28(17.4 \%)$ & 0.078 \\
\hline \multicolumn{5}{|l|}{ PCT (ng/mL),n(\%) } \\
\hline$\geq 0.5,<2$ & $90(29.9 \%)$ & $44(31.4 \%)$ & $46(28.6 \%)$ & 0.589 \\
\hline$\geq 2$ & $90(29.9 \%)$ & $49(35.0 \%)$ & $4 \mathrm{I}(25.5 \%)$ & 0.072 \\
\hline
\end{tabular}

Note: $* P<0.05$

Abbreviations: C. albicans, Candida albicans; NAC, non-albicans Candida; WBC, white blood count; NC, neutrophil count; IQR, interquartile range; LC, Lymphocyte count; NLR, neutrophil to lymphocyte ratio; TB, total bilirubin; AST, aspartate aminotransferase; ALT, alanine aminotransferase; PCT, procalcitonin.

a higher risk of $C$. albicans candidemia than NAC candidemia (aOR, 2.267; 95\% CI, 1.186-4.334).

\section{Species Distribution}

A total of 301 patients with candidemia were recruited in the current study, and C. albicans and NAC were responsible for $46.5 \%$ and $53.5 \%$ of candidemia cases, respectively. In NAC candidemia, the main species isolated were C. tropicalis, C. parapsilosis and C. glabrata, accounting for $23.9 \%, 15.6 \%$, and $10.3 \%$ of the cases, respectively. In 12 hematological malignancy patients with candidemia, more than $90 \%$ of the 
Table 3 Multivariable Logistic Regression of Risk Factors Caused by C. albicans vs NAC Candidemia

\begin{tabular}{|c|c|c|c|c|}
\hline Variables & Unadjusted OR $(95 \% \mathrm{Cl})$ & $P$-value & Adjusted OR (95\% Cl) & $P$-value \\
\hline Age ( $\geq 60$ years) & $1.726(1.058,2.813)$ & $0.029 *$ & I.522(0.878,2.638) & 0.135 \\
\hline Medical ward & $0.485(0.246,0.958)$ & $0.037^{*}$ & $\mathrm{I} .825(0.688,4.84 \mathrm{I})$ & 0.226 \\
\hline Surgical ward & $1.865(1.062,3.276)$ & $0.030 *$ & $1.853(0.929,3.694)$ & 0.080 \\
\hline Haematological malignancy & $0.098(0.013,0.770)$ & $0.027^{*}$ & $0.318(0.034,2.964)$ & 0.315 \\
\hline Diabetes mellitus & $2.138(1.174,3.895)$ & $0.013^{*}$ & $2.267(1.186,4.334)$ & $0.013^{*}$ \\
\hline Gastrointestinal disease & I.779(I.09I,2.902) & $0.021^{*}$ & $0.834(0.417,1.669)$ & 0.608 \\
\hline Surgery & $2.36 \mathrm{I}(\mathrm{I} .475,3.780)$ & $0.000 * *$ & $1.621(0.915,2.872)$ & 0.098 \\
\hline Abdominal surgery & $3.130(1.783,5.495)$ & $0.000 * *$ & $1.468(0.6|4,3.5| 3)$ & 0.388 \\
\hline Prior antifungal exposure & $0.316(0.123,0.810)$ & $0.016^{*}$ & $0.312(0.113,0.859)$ & $0.024 *$ \\
\hline Presence of CVC & $2.632(I .50 I, 4.615)$ & $0.001 *$ & $\mathrm{I} .882(0.872,4.06 \mathrm{I})$ & 0.107 \\
\hline Presence of PICC & $0.363(0.191,0.691)$ & $0.002 *$ & $0.696(0.32 \mathrm{I}, 1.507)$ & 0.358 \\
\hline Presence of arterial catheter & I.776(I.09I,2.889) & $0.02 I^{*}$ & $1.600(0.904,2.832)$ & 0.107 \\
\hline Presence of abdominal drainage tube & $2.993(1.688,5.307)$ & $0.000 * *$ & $1.594(0.679,3.744)$ & 0.284 \\
\hline
\end{tabular}

Notes: $* P<0.05, * * P<0.001$

Abbreviations: C. albicans, Candida albicans; NAC, non-albicans Candida; CVC, central venous catheter; PICC, peripherally inserted central catheter; OR, odds ratio; CI, confidence interval.

cases (11/12) were caused by NAC, especially C. tropicalis $(10 / 12,83.3 \%)$. The distribution of Candida spp. is shown in Table 4 and Figure 2.

\section{In vitro Susceptibilities}

As shown in Tables 5 and 6, NAC isolates had a significantly higher resistance to fluconazole, voriconazole, itraconazole and clotrimazole (all $\mathrm{P}<0.05$ ). In particularly, C. tropicalis had high resistance rates to clotrimazole (68.6\%), itraconazole (45.6\%), fluconazole $(50.0 \%)$, and voriconazole $(56.5 \%)$, whereas less than $3 \%$ of C. albicans isolates were resistant to these four drugs. Both C. albicans and NAC had a low resistance rate (less than $2.0 \%$ of isolates) to amphotericin B (Table 5).

In general, the resistance rate to ketoconazole (26.6\%) was the highest, followed by clotrimazole $(23.5 \%)$, fluconazole (14.5\%), and voriconazole (13.1\%) (Table 7). In terms of specific azoles, they had different resistance rates which were dependent on the different species of Candida. C. albicans was sensitive to azoles, but this was apparently not the case for NAC, as most of them were resistant to these azoles such as fluconazole, voriconazole and clotrimazole, with a high resistance rate of more than $50 \%$ (Table 7). Of note, $14.6 \%$ (44/301) of patients with candidemia exhibited cross-resistance, especially in patients with $C$. tropicalis among which the cross-resistance rate to azoles was as high as $50.0 \%(36 / 72)$. Among hematologic malignancy patients with $C$. tropicalis candidemia, the cross-resistance rate was up to $90 \%(9 / 10)$ (Table 4).

\section{Clinical Therapy}

The details about clinical features and treatments at the onset of candidemia are shown in Table 6, which indicated significant differences in RRT, source of infection (intraabdominal), and antifungal therapy between the two types of candidemia. A total of $10.6 \%$ of patients with NAC received RRT, which was almost three times that of patients with $C$. albicans $(P=0.020)$. The main identified sources of candidemia was catheter-related candidemia $(33.2 \%, 100 /$ $301)$ and intra-abdominal infections (13.0\%, 39/301), whereas $42.9 \%(129 / 301)$ of candidemia cases were

Table 4 Distribution of Candida spp., Cross-Resistance and Prior Antifungal Exposure of Patients with Hematological Malignancy

\begin{tabular}{|l|l|l|}
\hline Candida Species & Prior Antifungal Exposure $\mathbf{n}(\%)$ & Cross-Resistance $^{\mathbf{a}} \mathbf{n}(\%)$ \\
\hline C. albicans $(\mathrm{n}=\mathrm{I})$ & $0(0.0 \%)$ & $0(0.0 \%)$ \\
NAC $(\mathrm{n}=\mathrm{II})$ & $4(36.4 \%)$ & $10(90.9 \%)$ \\
C. tropicalis $(\mathrm{n}=10)$ & $3(30.0 \%)$ & $9(90.0 \%)$ \\
C. krusei $(\mathrm{n}=\mathrm{I})$ & $1(100.0 \%)$ & $1(100.0 \%)$ \\
\hline
\end{tabular}

Notes: ${ }^{a}$ Cross-resistance was defined as resistance to any two or more azoles in the drug sensitivity tests in this study.

Abbreviations: C. albicans, Candida albicans; NAC, non-albicans Candida; C. tropicalis, Candida tropicalis; C. krusei, Candida krusei. 


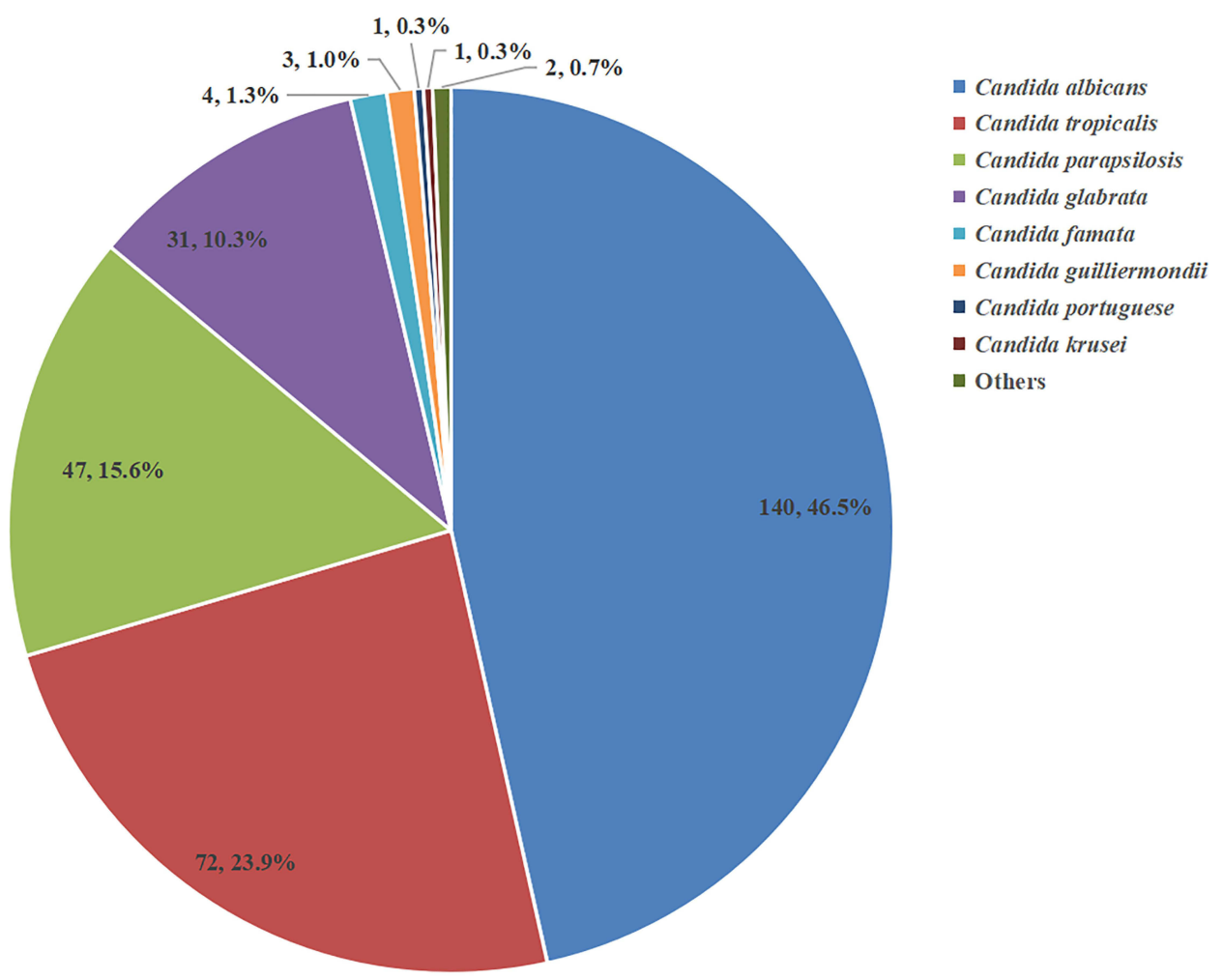

Figure 2 Distribution of different Candida spp. during this candidemia study period.

considered primary infections, as no obvious infection sources were confirmed. In further comparison, patients with NAC candidemia had fewer intraperitoneal sources for candidemia than patients with $C$. albicans candidemia (9.3\% vs $17.1 \%, P=0.044)$. In terms of source control, the percentage of catheter removal within $48 \mathrm{~h}$ in all patients with indwelling intravascular catheters was $73.0 \%$ (73/ 100), although no significant difference was found between these two groups. Regarding adequate antifungal treatment, the ratio of patients with candidemia was similar in both groups ( $31.7 \%$ vs $34.1 \%, P>0.05)$. In addition, pyrrole antifungal agents were more commonly used in patients with NAC, while echinocandin antifungal agents were more frequently given to patients with C. albicans (Table 6).

\section{Outcomes}

In patients with candidemia, the ICU length of stay was 14 days (IQR, 1.0-38.0), and the total length of hospitalization was 35 days (IQR, 19.3-65.0) (Table 8). Patients with NAC candidemia had a longer ICU stay and a longer total hospitalization than those with $C$. albicans candidemia [median days, $15.0(0.5-46.0)$ vs $14.0(2.0-33.8), P=0.406 ; 37.5$ (20.2-70.0) vs $34.0(19.0-60.0), P=0.303]$, but these values were not statistically significant. Furthermore, no significant differences were noted in the mortality rates at 14 days, 28 days and 60 days between these two groups, which was consistent with the result of survival curve (Figure 3).

\section{Discussion}

Several findings have been revealed in our current study. First, although $C$. albicans was reported to be the major fungal species, NAC spp. accounted for 53.5\% (161/301) of candidemia. Second, several risk factors for NAC candidemia were found, including medical admission, hematological malignancies, prior antifungal exposure, and the presence of PICC. Particularly, prior antifungal exposure constituted one of the most pivotal independent risk factors for NAC candidemia, which differed from that of diabetes mellitus for C. albicans candidemia. Moreover, C. albicans remained highly susceptible to most antifungal agents (including azoles), whereas NAC showed strikingly different responses to azoles, especially C. tropicalis, which had a high cross-resistance to azoles. Lastly, no significant differences in the clinical outcomes were observed between these two groups.

To date, numerous studies have specifically described the epidemiology of candidemia based on demographic surveys from around the world..$^{7-11,20-22}$ As expected, the 
Table 5 Comparison of Antifungal Susceptibilities of Different Candida spp. in vitro

\begin{tabular}{|c|c|c|c|c|}
\hline Species (n) & Antifungal Agent & $\mathbf{S}, \mathbf{n}(\%)$ & I, n(\%) & $\mathbf{R}, \mathbf{n}(\%)$ \\
\hline \multirow[t]{8}{*}{ C. albicans $(n=140)$} & 5-fluorocytosine & $64(98.5 \%)$ & $0(0.0 \%)$ & $\mathrm{I}(\mathrm{I} .5 \%)$ \\
\hline & Fluconazole & $123(96.1 \%)$ & $4(3.1 \%)$ & $\mathrm{I}(0.8 \%)$ \\
\hline & Amphotericin B & $134(100.0 \%)$ & $0(0.0 \%)$ & $0(0.0 \%)$ \\
\hline & Voriconazole & 124(99.2\%) & $0(0.0 \%)$ & $\mathrm{I}(0.8 \%)$ \\
\hline & Itraconazole & $128(95.6 \%)$ & $3(2.2 \%)$ & $3(2.2 \%)$ \\
\hline & Clotrimazole & $66(98.5 \%)$ & $0(0.0 \%)$ & $\mathrm{I}(\mathrm{I} .5 \%)$ \\
\hline & Ketoconazole & $25(42.4 \%)$ & $21(35.6 \%)$ & $13(22.0 \%)$ \\
\hline & Nystatin & $66(98.5 \%)$ & $0(0.0 \%)$ & $\mathrm{I}(\mathrm{I} .5 \%)$ \\
\hline \multicolumn{5}{|l|}{$\operatorname{NAC}(n=161)$} \\
\hline \multirow[t]{8}{*}{ C. tropicalis $(n=72)$} & 5-fluorocytosine & $36(100.0 \%)$ & $0(0.0 \%)$ & $0(0.0 \%)$ \\
\hline & Fluconazole & $31(44.3 \%)$ & $4(5.7 \%)$ & $35(50.0 \%)$ \\
\hline & Amphotericin B & $7 \mathrm{I}(98.6 \%)$ & $0(0.0 \%)$ & $\mathrm{I}(\mathrm{I} .4 \%)$ \\
\hline & Voriconazole & $27(43.5 \%)$ & $0(0.0 \%)$ & $35(56.5 \%)$ \\
\hline & Itraconazole & $29(42.6 \%)$ & $8(11.8 \%)$ & $3 I(45.6 \%)$ \\
\hline & Clotrimazole & $12(23.5 \%)$ & $4(7.8 \%)$ & $35(68.6 \%)$ \\
\hline & Ketoconazole & $9(26.5 \%)$ & $13(38.2 \%)$ & $12(35.3 \%)$ \\
\hline & Nystatin & $32(97.0 \%)$ & I(3.0\%) & $0(0.0 \%)$ \\
\hline \multirow[t]{8}{*}{ C. parapsilosis $(n=47)$} & 5-fluorocytosine & $24(100.0 \%)$ & $0(0.0 \%)$ & $0(0.0 \%)$ \\
\hline & Fluconazole & $42(93.3 \%)$ & $2(4.4 \%)$ & $I(2.2 \%)$ \\
\hline & Amphotericin B & $24(100.0 \%)$ & $0(0.0 \%)$ & $0(0.0 \%)$ \\
\hline & Voriconazole & $4 I(97.6 \%)$ & $0(0.0 \%)$ & $\mathrm{I}(2.4 \%)$ \\
\hline & Itraconazole & $38(92.7 \%)$ & $3(7.3 \%)$ & $0(0.0 \%)$ \\
\hline & Clotrimazole & $19(90.5 \%)$ & $\mathrm{I}(4.8 \%)$ & $\mathrm{I}(4.8 \%)$ \\
\hline & Ketoconazole & $10(50.0 \%)$ & $6(30.0 \%)$ & $4(20.0 \%)$ \\
\hline & Nystatin & $20(100.0 \%)$ & $0(0.0 \%)$ & $0(0.0 \%)$ \\
\hline \multirow[t]{8}{*}{ C. glabrata $(\mathrm{n}=31)$} & 5-fluorocytosine & $9(100.0 \%)$ & $0(0.0 \%)$ & $0(0.0 \%)$ \\
\hline & Fluconazole & $24(80.0 \%)$ & $4(\mid 3.3 \%)$ & $2(6.7 \%)$ \\
\hline & Amphotericin B & $31(100.0 \%)$ & $0(0.0 \%)$ & $0(0.0 \%)$ \\
\hline & Voriconazole & $25(96.2 \%)$ & $0(0.0 \%)$ & $\mathrm{I}(3.8 \%)$ \\
\hline & Itraconazole & $14(56.0 \%)$ & $6(24.0 \%)$ & $5(20.0 \%)$ \\
\hline & Clotrimazole & $14(73.7 \%)$ & $3(15.8 \%)$ & $2(10.5 \%)$ \\
\hline & Ketoconazole & $8(42.1 \%)$ & $4(21.1 \%)$ & $7(36.8 \%)$ \\
\hline & Nystatin & $22(100.0 \%)$ & $0(0.0 \%)$ & $0(0.0 \%)$ \\
\hline \multirow[t]{8}{*}{ Other Candida spp. ${ }^{\mathrm{a}}(\mathrm{n}=\mathrm{II})$} & 5-fluorocytosine & $3(100.0 \%)$ & $0(0.0 \%)$ & $0(0.0 \%)$ \\
\hline & Fluconazole & $7(70.0 \%)$ & $I(I 0.0 \%)$ & $2(20.0 \%)$ \\
\hline & Amphotericin B & $\mathrm{II}(100.0 \%)$ & $0(0.0 \%)$ & $0(0.0 \%)$ \\
\hline & Voriconazole & $8(100.0 \%)$ & $0(0.0 \%)$ & $0(0.0 \%)$ \\
\hline & Itraconazole & $9(90.0 \%)$ & $\mathrm{I}(\mathrm{I} 0.0 \%)$ & $0(0.0 \%)$ \\
\hline & Clotrimazole & $8(100.0 \%)$ & $0(0.0 \%)$ & $0(0.0 \%)$ \\
\hline & Ketoconazole & $6(75.0 \%)$ & $\mathrm{I}(\mathrm{I} 2.5 \%)$ & $\mathrm{I}(12.5 \%)$ \\
\hline & Nystatin & $8(100.0 \%)$ & $0(0.0 \%)$ & $0(0.0 \%)$ \\
\hline
\end{tabular}

Notes: Not all agents listed have been tested in all isolated species. ${ }^{a}$ Included Candida famata $(\mathrm{n}=4)$, Candida guilliermondii $(\mathrm{n}=3)$, Candida $(\mathrm{n}=2)$, Candida portuguese $(\mathrm{n}$ $=1$ ), and Candida krusei $(\mathrm{n}=\mathrm{I})$.

Abbreviations: S, susceptible; I, intermediate; R, resistant; $C$. albicans, Candida albicans; NAC, non-albicans Candida; $C$. tropicalis, Candida tropicalis; $C$. parapsilosis, Candida parapsilosis; C. glabrata, Candida glabrata.

four major pathogens of candidemia were C. albicans, C. tropicalis, C. glabrata and C. parapsilosis, which accounted for $96.3 \%$ of all Candida spp. in this study (Figure 2). NAC spp. collectively represented $53.5 \%$ of the bloodstream isolates, which exceeded the percent of C. albicans spp., and these results are consistent with the results from Northeast China, Latin America, North America and Asia-Pacific. ${ }^{7,10,23}$ Concerning NAC 
Table 6 Clinical Features and Treatments of Patients with C. albicans or NAC at the Onset of Candidemia

\begin{tabular}{|c|c|c|c|c|}
\hline Clinical Features and Treatments & Total $(n=30 I)$ & C. albicans $(n=140)$ & NAC $(n=161)$ & $P$-value \\
\hline Septic shock, n(\%) & $\mathrm{III}(36.8 \%)$ & $5 \mathrm{I}(36.4 \%)$ & $60(37.3 \%)$ & 0.880 \\
\hline Fluid resuscitation, n(\%) & $70(23.3 \%)$ & $30(21.4 \%)$ & $40(24.8 \%)$ & 0.484 \\
\hline Vasopressor therapy, n(\%) & $96(31.9 \%)$ & $45(32.1 \%)$ & $5 \mathrm{I}(3 \mathrm{I} .7 \%)$ & 0.931 \\
\hline RRT, n(\%) & $22(7.3 \%)$ & $5(3.6 \%)$ & $17(10.6 \%)$ & $0.020 *$ \\
\hline Hydrocortisone treatment, $n(\%)$ & $4(1.3 \%)$ & $\mathrm{I}(0.7 \%)$ & $3(1.9 \%)$ & 0.385 \\
\hline Concomitant bacterial infection, $n(\%)$ & $58(19.3 \%)$ & $25(17.9 \%)$ & $33(20.5 \%)$ & 0.562 \\
\hline \multicolumn{5}{|l|}{ Source of candidemia } \\
\hline Catheter-related candidemia, $n(\%)$ & $100(33.2 \%)$ & $45(32.1 \%)$ & $55(34.2 \%)$ & 0.711 \\
\hline Pulmonary infection, $n(\%)$ & $14(4.7 \%)$ & $9(6.4 \%)$ & $5(3.1 \%)$ & 0.172 \\
\hline Urinary tract infection, $\mathrm{n}(\%)$ & $13(4.3 \%)$ & $7(5 \%)$ & $6(3.7 \%)$ & 0.588 \\
\hline Intra-abdominal infection, $\mathrm{n}(\%)$ & $39(13.0 \%)$ & $24(17.1 \%)$ & $15(9.3 \%)$ & $0.044 *$ \\
\hline Others ${ }^{\mathrm{a}}, \mathrm{n}(\%)$ & $129(42.9 \%)$ & $52(37.1 \%)$ & $77(47.8 \%)$ & 0.062 \\
\hline Remove the catheters $(\leq 48 \mathrm{~h})(45 \text { vs } 55)^{\mathrm{b}}, \mathrm{n}(\%)$ & $73(73.0 \%)$ & $36(80.0 \%)$ & $37(67.3 \%)$ & 0.154 \\
\hline Adequate antifungal treatment, $n(\%)$ & $99(32.9 \%)$ & $48(34.1 \%)$ & $5 \mathrm{I}(3 \mathrm{I} .7 \%)$ & 0.631 \\
\hline Antifungal therapy + catheter removal ( $\leq 48 \mathrm{~h})(36 \text { vs } 37)^{\mathrm{c}}, \mathrm{n}(\%)$ & $38(52.1 \%)$ & $21(58.3 \%)$ & $17(45.9 \%)$ & 0.290 \\
\hline \multicolumn{5}{|l|}{ Antifungal agents } \\
\hline Pyrroles, $\mathrm{n}(\%)$ & $167(55.5 \%)$ & $72(51.4 \%)$ & $95(59.0 \%)$ & 0.187 \\
\hline Echinocandins, $n(\%)$ & $|4|(46.8 \%)$ & $66(47.1 \%)$ & $75(46.6 \%)$ & 0.923 \\
\hline Duration of antifungal therapy, median days (IQR) & $9.0(4.0,16.5)$ & $9.0(4.0,16.0)$ & $10.0(4.0,17.0)$ & 0.544 \\
\hline \multicolumn{5}{|l|}{ Antifungal resistance ${ }^{d}$} \\
\hline 5-fluorocytosine (65 vs 72$)^{\mathrm{e}}, \mathrm{n}(\%)$ & $\mathrm{I}(0.7 \%)$ & $\mathrm{I}(1.5 \%)$ & $0(0.0 \%)$ & 0.959 \\
\hline Fluconazole (I28 vs 155$)^{\mathrm{e}}, \mathrm{n}(\%)$ & $4 \mathrm{I}(14.5 \%)$ & $\mathrm{I}(0.8 \%)$ & $40(25.8 \%)$ & $0.000 * *$ \\
\hline Amphotericin B (134 vs I38) ${ }^{\mathrm{e}}, \mathrm{n}(\%)$ & $\mathrm{I}(0.4 \%)$ & $0(0.0 \%)$ & $\mathrm{I}(0.7 \%)$ & 1.000 \\
\hline Voriconazole (1 25 vs 138$)^{\mathrm{e}}, \mathrm{n}(\%)$ & $38(14.4 \%)$ & $\mathrm{I}(0.8 \%)$ & $37(26.8 \%)$ & $0.000 * *$ \\
\hline Itraconazole (|34 vs |44) ${ }^{\mathrm{e}}, \mathrm{n}(\%)$ & $39(14.0 \%)$ & $3(2.2 \%)$ & $36(25.0 \%)$ & $0.000 * *$ \\
\hline Clotrimazole (67 vs 99) $)^{\mathrm{e}}, \mathrm{n}(\%)$ & $39(23.4 \%)$ & $\mathrm{I}(\mathrm{I} .5 \%)$ & $38(38.4 \%)$ & $0.000 * *$ \\
\hline Ketoconazole (59 vs 81$)^{\mathrm{e}}, \mathrm{n}(\%)$ & $37(26.4 \%)$ & $13(22.0 \%)$ & $24(29.6 \%)$ & 0.314 \\
\hline Nystatin $(67 \text { vs } 83)^{\mathrm{e}}, \mathrm{n}(\%)$ & $2(1.3 \%)$ & $\mathrm{I}(\mathrm{I} .5 \%)$ & $\mathrm{I}(\mathrm{I} .2 \%)$ & 0.914 \\
\hline
\end{tabular}

Notes: $* P<0.05, * * P<0.001$. ${ }^{a}$ The source of infection could not be identified or primary infection. ${ }^{\mathrm{b}}$ The numbers in parentheses represented the total numbers of Candida spp. with intravascular catheters. ${ }^{C}$ The numbers in parentheses represented the total numbers of Candida spp. with catheter removal. ${ }^{d} \mathrm{Not}$ all agents listed have been tested in all isolates. ${ }^{\mathrm{e}}$ The numbers in parentheses represented the total numbers of Candida species performed susceptibility test.

Abbreviations: C. albicans, Candida albicans; NAC, non-albicans Candida; RRT, renal replacement therapy; IQR, interquartile range.

candidemia, it is worth noting that $C$. tropicalis has become a common NAC spp. worldwide. ${ }^{7,8}$ In our study, C. tropicalis ranked second among all Candida spp. in candidemia, accounting for $23.9 \%$. This rate was lower than the rate in Asia-Pacific (30.7\%), but higher than that in Northeast China (10\%), Latin America (17.0\%), North America (8.0\%) and Europe (7.5\%). ${ }^{7,10,23}$ The epidemiological difference in species for candidemia might vary with patient age, geographical area, medical practice and use of antifungal drugs. ${ }^{1}$ The prevalence of NAC candidemia has significantly increased over time, which is generally associated with a reduced antifungal sensitivity resulting from the widespread use of azoles..$^{7,8,24,25}$ Other possible explanations may include the increased number of immunocompromised patients, the growing use of invasive medical procedures, and the improvement of yeast isolation techniques at the species level. 1,11,22 However, the underlying mechanisms causing the epidemiological changes of NAC spp. in candidemia remain uncertain.

Common risk factors for NAC candidemia consisted of medical admissions, hematological malignancies, antifungal exposure, and the presence of PICC (Table 1). Other studies 
Table 7 In vitro Drug Resistance of Candida spp. to Azoles

\begin{tabular}{|c|c|c|c|c|c|c|}
\hline \multirow[t]{2}{*}{ Species (n) } & Fluconazole & Voriconazole & Itraconazole & Clotrimazole & Ketoconazole & Cross-Resistance $^{a}$ \\
\hline & $\mathbf{R}, \mathbf{n}(\%)$ & $R, n(\%)$ & $R, n(\%)$ & $R, n(\%)$ & $\mathbf{R}, \mathbf{n}(\%)$ & $n(\%)$ \\
\hline C. albicans $(n=140)$ & $\mathrm{I}(0.8 \%)$ & $\mathrm{I}(0.8 \%)$ & $3(2.2 \%)$ & $\mathrm{I}(\mathrm{I} .5 \%)$ & $13(22.0 \%)$ & $3(2.1 \%)$ \\
\hline \multicolumn{7}{|l|}{$\operatorname{NAC}(n=161)$} \\
\hline C. tropicalis $(n=72)$ & $35(50.0 \%)$ & $35(56.5 \%)$ & $31(45.6 \%)$ & $35(68.6 \%)$ & $12(35.3 \%)$ & $36(50.0 \%)$ \\
\hline C. parapsilosis $(n=47)$ & $\mathrm{I}(2.2 \%)$ & $\mathrm{I}(2.4 \%)$ & $0(0.0 \%)$ & $\mathrm{I}(4.8 \%)$ & $4(20.0 \%)$ & $\mathrm{I}(2.1 \%)$ \\
\hline C. glabrata $(\mathrm{n}=3 \mathrm{I})$ & $2(6.7 \%)$ & $\mathrm{I}(3.8 \%)$ & $5(20.0 \%)$ & $2(10.5 \%)$ & $7(36.8 \%)$ & $3(9.7 \%)$ \\
\hline Other species $^{\mathrm{b}}(\mathrm{n}=\mathrm{II})$ & $2(20.0 \%)$ & $0(0.0 \%)$ & $0(0.0 \%)$ & $0(0.0 \%)$ & $\mathrm{I}(\mathrm{I} 2.5 \%)$ & $I^{c}(9.1 \%)$ \\
\hline Total $(n=301)$ & $4 \mathrm{I}(14.5 \%)$ & $38(13.1 \%)$ & II(4.0\%) & $39(23.5 \%)$ & $37(26.6 \%)$ & $44(\mid 4.6 \%)$ \\
\hline
\end{tabular}

Notes: Not all agents listed have been tested in all isolated species. ${ }^{\mathrm{a} C}$ ross-resistance was defined as resistance to any two or more of the above azoles. ${ }^{\mathrm{b}}$ Included Candida famata $(n=4)$, Candida guilliermondii $(n=3)$, Candida $(n=2)$, Candida portuguese $(n=1)$, and Candida krusei $(n=1)$. ${ }^{c}$ Only one case of Candida krusei had cross-resistance in other Candida species.

Abbreviations: R, resistant; C. albicans, Candida albicans; NAC, non-albicans Candida; C. tropicalis, Candida tropicalis; C. parapsilosis, Candida parapsilosis; C. glabrata, Candida glabrata.

Table 8 Outcomes of Patients with C. albicans and NAC Candidemia

\begin{tabular}{|l|l|l|l|l|}
\hline Outcomes & Total (n=30I) & C. albicans (n=I 40) & NAC (n=I6I) & P -value \\
\hline Length of ICU stay (M) (IQR) & $14.0(1.0,38.0)$ & $14.0(2.0,33.8)$ & $15.0(0.5,46.0)$ & 0.406 \\
Length of hospital stay (M) (IQR) & $35.0(19.3,65.0)$ & $34.0(19.0,60.0)$ & $37.5(20.2,70.0)$ & 0.303 \\
Crude I4-day mortality, n(\%) & $87(28.9 \%)$ & $44(31.4 \%)$ & $43(26.7 \%)$ & 0.368 \\
Crude 28-day mortality, n(\%) & $104(34.6 \%)$ & $53(37.9 \%)$ & $51(31.7 \%)$ & 0.261 \\
Crude 60-day mortality, n(\%) & $114(37.9 \%)$ & $58(41.4 \%)$ & $56(34.8 \%)$ & 0.236 \\
Crude in-hospital mortality, n(\%) & $122(40.5 \%)$ & $62(44.3 \%)$ & $60(37.3 \%)$ & 0.216 \\
\hline
\end{tabular}

Abbreviations: C. albicans, Candida albicans; NAC, non-albicans Candida; ICU, intensive care unit; M, median; IQR, interquartile range.

have found that hematological malignancies and prior exposure to antifungal agents were factors closely related to NAC candidemia in comparison with $C$. albicans candidemia, ${ }^{26-}$ ${ }^{29}$ which echoes our study. Among hematological malignancy patients with candidemia, NAC was the main type, in which $C$. tropicalis accounted for $90.9 \%$ (10/11) (Table 4). Other studies also showed that $C$. tropicalis was the most common NAC spp. in hematological malignancy patients complicated with candidemia. ${ }^{27,30}$ This peculiar epidemiology might be explained by the increased invasiveness of $C$. tropicalis in the human gastrointestinal tract, especially in patients with hematological malignancies who are immunocompromised. ${ }^{31}$ Furthermore, a high proportion of antifungal exposure before the onset of candidemia (36.4\%) was observed in hematological malignancy patients with NAC candidemia, which might be partly responsible for the species' migration to NAC. However, hematological malignancy was not independently associated with NAC candidemia after the multivariate regression analysis (Table 3), possibly due to the low proportion of these patients in our study $(6.8 \%)$ (Table 1$)$. Of note, when these risk factors were further analyzed using the multivariate regression, prior antifungal exposure was independently associated with an increased risk of NAC candidemia, while diabetes mellitus was associated with an increased risk of C. albicans candidemia (Table 3). However, it remains unknown whether patients with both risk factors, diabetes and prior antifungal exposure, are likely to develop mixed BSIs of $C$. albicans and NAC, which merits further investigation.

Over the past 20 years, the drug resistance of Candida to azoles has attracted worldwide attention. Although azoles show preliminary clinical benefits in C. albicans candidemia, ${ }^{7,8}$ the increasing prevalence of NAC spp. and their associated reduced antifungal sensitivity have become a main challenge in candidemia treatment. ${ }^{20,22,25}$ In the current study, NAC demonstrated significantly higher resistance to fluconazole, voriconazole, itraconazole and clotrimazole (all $\mathrm{P}<0.05$ ), especially with $C$. tropicalis isolates (Tables 6 and 7). We observed that the rate of azole resistance in C. tropicalis was over $35 \%$, which was consistent with the high resistance rate in the CHIF-NET study; ${ }^{8}$ Furthermore, $50 \%(36 / 72)$ of 


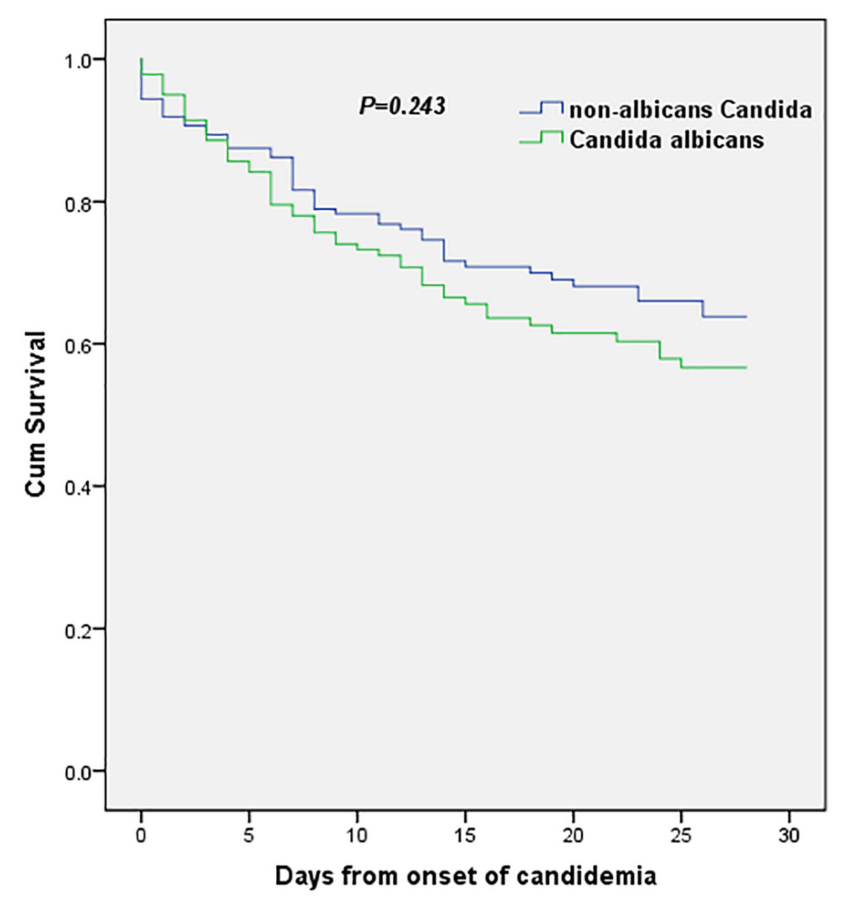

Figure 3 Kaplan-Meier estimates of survival in patients with C. albicans candidemia and NAC candidemia.

C. tropicalis isolates had cross-resistance to azoles. A striking result of this study was that the cross-resistance rate of C. tropicalis to azoles in hematological malignancy patients with candidemia was up to $90 \%$ (Table 4). Globally, the resistance to azoles in C. tropicalis mainly occurs in the AsiaPacific region, while it is still low (less than 10\%) in European and American countries. ${ }^{7,8,23}$ Previous works have reported several variables that might contribute to high azole resistance among NAC, such as prior exposure to antifungal drugs (especially azoles) or antibiotics, the duration of prior drug exposure or inappropriate dosing. ${ }^{30,32}$ Moreover, Fan et $\mathrm{al}^{33}$ showed that one explanation of the azole resistance in C. tropicalis isolates was the ERG11 missense mutations. Since $C$. tropicalis candidemia has been reported to have a higher mortality and a poor prognosis, ${ }^{34}$ we should highlight the importance of monitoring antifungal drug resistance in C. tropicalis infections. Additionally, echinocandins might be used as an initial treatment for patients who have some risk factors for NAC candidemia, according to the clinical guidelines recommended by the Infectious Diseases Society of America. ${ }^{19}$

In view of the high incidence of Candida and the high resistance of antifungal agents, it is important to develop new antifungal agents. In recent years, several studies have found that natural compounds have efficacy against Candida. $^{35-38}$ Some essential oils extracted from plants have displayed inhibitory effects on the growth and activity of common Candida isolates. ${ }^{35,36,38}$ UOST5-NPS, a novel anticandidal azole agent based on essential oils, has been developed for the newly emerged Candida auris. ${ }^{37}$ In addition, amphotericin B combined with Ruta graveolens essential oil has also shown synergistic effects against $C$. albicans and $C$. tropicalis in vitro. ${ }^{36}$ These results suggest that these natural compounds might provide a new promising strategy against Candida infection in the future.

Although some studies have reported worse outcomes for NAC candidemia in comparison with $C$. albicans candidemia, ${ }^{10,11}$ few significant differences were observed between these two groups in our current study. (Table 8, Figure 3). This might be partly due to the similar disease severity, similar baseline comorbidities (Table 1), and similar clinical treatments at the onset of candidemia (Table 6) between the two groups.

Notably, some limitations exist in the current study. First, our results were mainly from a single-center study; therefore, they may not represent the trends in the other regions of China. Nevertheless, these data could be used as a reference. Second, although a positive blood sample culture is the gold standard in the diagnosis of candidemia, many patients do not have a positive blood culture result due to its poor sensitivity. Thus, the diagnosis of candidemia might be underestimated. Finally, echinocandins were not included in the drug sensitivity tests in this study, as we could not detect the sensitivity to echinocandins. According to the CHIF-NET study, Candida spp. were highly sensitive to echinocandins in vitro. ${ }^{8}$

\section{Conclusion}

Together, we have revealed that NACs are dominant in candidemia in our current study. Several factors, including medical admissions, hematological malignancies, antifungal exposure, and the presence of PICC, are closely related to NAC candidemia, whereas prior antifungal exposure is an independent risk factor. Of note, although the outcomes of NAC and C. albicans candidemia are similar, drug resistance to specific azoles as well as cross-resistance frequently occurs in patients with NAC candidemia (especially $C$. tropicalis), and this deserves further evaluation in clinical practice and further in-depth investigations.

\section{Abbreviations}

(A) C. albicans, Candida albicans; NAC, non-albicans Candida; C. parapsilosis, Candida parapsilosis; C. tropicalis, Candida tropicalis; C. glabrata, Candida glabrata; spp., species; BSI, bloodstream 
infection; IQR, interquartile range; ICU, intensive care unit; CCI, Charlson Comorbidity Index; APACHE, acute physiology and chronic health evaluation; SOFA, sequential organ failure assessment; CRBSI, catheter-related bloodstream infection; GI, gastrointestinal; TPN, total parenteral nutrition; CVC, central venous catheter; PICC, peripherally inserted central catheter; WBC, white blood count; NC, neutrophil count; LC, lymphocyte count; NLR, neutrophil to lymphocyte ratio; TB, total bilirubin; AST, aspartate aminotransferase; ALT, alanine aminotransferase; PCT, procalcitonin; OR, odds ratio; CI, confidence interval; S, susceptible; I, intermediate; R, resistant; RRT, renal replacement therapy.

\section{Data Sharing Statement}

All data generated and/or analyzed during the current study are included in this manuscript.

\section{Ethics Approval and Informed Consent}

This study received human research ethics approval (NO. 2020-744) from the Ethics Committee of the Second Affiliated Hospital, Zhejiang University School of Medicine. We ensure the confidentiality of patient data and comply with the Helsinki statement. Due to the retrospective nature of the study, the Ethics Committee determined that no patient consent was required.

\section{Author Contributions}

All authors made a significant contribution to the work reported, whether that is in the conception, study design, execution, acquisition of data, analysis and interpretation, or in all these areas; took part in drafting, revising or critically reviewing the article; gave final approval of the version to be published; have agreed on the journal to which the article has been submitted; and agree to be accountable for all aspects of the work.

\section{Funding}

This work was supported in part by grants from National Natural Science Foundations of China (No. 81971871, GS Zhang), Natural Science Foundation of Zhejiang Province (No. LY19H150007, GS Zhang), and Medical and Health Research Program of Zhejiang Province (No. 2021KY174, GS Zhang).

\section{Disclosure}

The authors report no conflicts of interest in this work.

\section{References}

1. Pfaller MA, Diekema DJ. Epidemiology of invasive candidiasis: a persistent public health problem. Clin Microbiol Rev. 2007;20 (1):133-163. doi:10.1128/CMR.00029-06

2. Lausch KR, Søgaard M, Rosenvinge FS, et al. High incidence of candidaemia in a nationwide cohort: underlying diseases, risk factors and mortality. Int $J$ Infect Dis. 2018;76:58-63. doi:10.1016/j. ijid.2018.08.010

3. Colombo AL, Nucci M, Park BJ, et al. Epidemiology of candidemia in Brazil: a nationwide sentinel surveillance of candidemia in eleven medical centers. $J$ Clin Microbiol. 2006;44(8):2816-2823. doi:10.1128/JCM.00773-06

4. Jia X, Li C, Cao J, Wu X, Zhang L. Clinical characteristics and predictors of mortality in patients with candidemia: a Six-Year Retrospective Study. Eur J Clin Microbiol. 2018;37(9):1717-1724. doi:10.1007/s10096-018-3304-9

5. Cuervo G, Garcia-Vidal C, Puig-Asensio M, et al. Usefulness of guideline recommendations for prognosis in patients with candidemia. Med Mycol. 2019;57(6):659-667. doi:10.1093/mmy/ myy 118

6. Lee RA, Zurko JC, Camins BC, et al. Impact of infectious disease consultation on clinical management and mortality in patients with candidemia. Clin Infect Dis. 2019;68(9):1585-1587. doi:10.1093/cid/ ciy849

7. Pfaller MA, Diekema DJ, Turnidge JD, Castanheira M, Jones RN. Twenty years of the SENTRY antifungal surveillance program: results for candida species from 1997-2016. Open Forum Infect Dis. 2019;6(Suppl 1):S79-S94. doi:10.1093/ofid/ofy358

8. Xiao M, Chen SC, Kong F, et al. Distribution and antifungal susceptibility of candida species causing candidemia in china: an update from the CHIF-NET Study. J Infect Dis. 2020;221(Suppl 2):S139S147. doi:10.1093/infdis/jiz573

9. Chi H, Yang Y, Shang S, et al. Candida albicans versus non-albicans bloodstream infections: the comparison of risk factors and outcome. J Microbiol Immunol Infect. 2011;44(5):369-375. doi:10.1016/j. jmii.2010.08.010

10. Zhang W, Song X, Wu H, Zheng R. Epidemiology, risk factors and outcomes of Candida albicans vs. non- albicans candidaemia in adult patients in Northeast China. Epidemiol Infect. 2019;147:e277-e277. doi:10.1017/s0950268819001638

11. Dimopoulos G, Ntziora F, Rachiotis G, Armaganidis A, Falagas ME. Candida albicans versus non-albicans intensive care unit-acquired bloodstream infections: differences in risk factors and outcome. Anesth Analg. 2008;106(2):523-529. doi:10.1213/ ane. $0 \mathrm{~b} 013 \mathrm{e} 3181607262$

12. Zhong L, Zhang S, Tang K, et al. Clinical characteristics, risk factors and outcomes of mixed Candida albicans/bacterial bloodstream infections. Bmc Infect Dis. 2020;20(1):1-810. doi:10.1186/s12879020-05536-z

13. Clinical and Laboratory Standards Institute. M27-A3. Reference Method for Broth Dilution Antifungal Susceptibility Testing of Yeasts, Approved Standard. 3rd ed. Wayne, PA: CLSI; 2008.

14. Clinical and Laboratory Standards Institute. M60. Performance Standards for Antifungal Susceptibility Testing of Yeasts. 1st ed. Wayne, PA: CLSI; 2018.

15. Mermel LA, Allon M, Bouza E, et al. Clinical practice guidelines for the diagnosis and management of intravascular catheter-related infection: 2009 update by the infectious diseases society of America. Clin Infect Dis. 2009;49(1):1-45. doi:10.1086/599376 
16. Seymour CW, Liu VX, Iwashyna TJ, et al. Assessment of clinical criteria for sepsis. JAMA. 2016;315(8):762. doi:10.1001/ jama.2016.0288

17. Kim SH, Yoon YK, Kim MJ, Sohn JW. Risk factors for and clinical implications of mixed Candida/bacterial bloodstream infections. Clin Microbiol Infect. 2013;19(1):62-68. doi:10.1111/j.14690691.2012.03906.x

18. Garnacho-Montero J, Diaz-Martin A, Garcia-Cabrera E, Ruiz Perez de Pipaon M, Hernandez-Caballero C, Lepe-Jimenez JA. Impact on hospital mortality of catheter removal and adequate antifungal therapy in Candida spp. bloodstream infections. J Antimicrob Chemother. 2012;68(1):206-213. doi:10.1093/jac/dks347

19. Pappas PG, Kauffman CA, Andes DR, et al. Clinical practice guideline for the management of candidiasis: 2016 update by the infectious diseases society of America. Clin Infect Dis. 2016;62(4):e1-e50. doi: $10.1093 / \mathrm{cid} / \mathrm{civ} 933$

20. Lamoth F, Lockhart SR, Berkow EL, Calandra T. Changes in the epidemiological landscape of invasive candidiasis. J Antimicrob Chemother. 2018;73(suppl 1):i4-i13. doi:10.1093/jac/dkx444

21. Song Y, Chen X, Yan Y, Wan Z, Liu W, Li R. Prevalence and antifungal susceptibility of pathogenic yeasts in china: a 10-Year Retrospective Study in a teaching hospital. Front Microbiol. 2020;11:1410. doi:10.3389/fmicb.2020.01401

22. Pappas PG, Lionakis MS, Arendrup MC, Ostrosky-Zeichner L, Kullberg BJ. Invasive candidiasis. Nat Rev Dis Primers. 2018;4 (1):18026. doi:10.1038/nrdp.2018.26

23. Tan TY, Hsu LY, Alejandria MM, et al. Antifungal susceptibility of invasive Candida bloodstream isolates from the Asia-Pacific region. Med Mycol. 2016;54(5):471-477. doi:10.1093/mmy/ myv114

24. Enoch DA, Yang H, Aliyu SH, Micallef C. The changing epidemiology of invasive fungal infections. Methods Mol Biol. 2017;1508:17-65. doi:10.1007/978-1-4939-6515-1_2

25. Perlin DS, Rautemaa-Richardson R, Alastruey-Izquierdo A. The global problem of antifungal resistance: prevalence, mechanisms, and management. Lancet Infect Dis. 2017;17(12):e383-e392. doi:10.1016/S1473-3099(17)30316-X

26. Apisarnthanarak A, Naknarongkij N, Kiratisin P, Mundy LM. Risk factors and outcomes of Candida albicans and non-albicans Candida species at a Thai tertiary care center. Am J Infect Control. 2009;37 (9):781-782. doi:10.1016/j.ajic.2009.04.289

27. Vigouroux S, Morin O, Moreau P, Harousseau JL, Milpied N. Candidemia in patients with hematologic malignancies: analysis of 7 years' experience in a single center. Haematologica. 2006;91 (5):717-718
28. Horn DL, Neofytos D, Anaissie EJ, et al. Epidemiology and outcomes of candidemia in 2019 patients: data from the prospective antifungal therapy alliance registry. Clin Infect Dis. 2009;48 (12):1695-1703. doi:10.1086/599039

29. Viscoli C, Girmenia C, Marinus A, et al. Candidemia in cancer patients: a prospective, multicenter surveillance study by the Invasive Fungal Infection Group (IFIG) of the European Organization for Research and Treatment of Cancer (EORTC). Clin Infect Dis. 1999;28(5):1071-1079. doi:10.1086/514731

30. Chen XC, Xu J, Wu DP. Clinical characteristics and outcomes of breakthrough candidemia in 71 hematologic malignancy patients and/ or allogeneic hematopoietic stem cell transplant recipients: a SingleCenter Retrospective Study from China, 2011-2018. Clin Infect Dis. 2020;71(Supp14):S394-S399. doi:10.1093/cid/ciaa1523

31. Walsh TJ, Merz WG. Pathologic features in the human alimentary tract associated with invasiveness of Candida tropicalis. Am J Clin Pathol. 1986;85(4):498-502. doi:10.1093/ajcp/85.4.498

32. Ben-Ami R, Olshtain-Pops K, Krieger M, et al. Antibiotic exposure as a risk factor for fluconazole-resistant Candida bloodstream infection. Antimicrob Agents Chemother. 2012;56(5):2518-2523. doi:10.1128/AAC.05947-11

33. Fan X, Xiao M, Zhang D, et al. Molecular mechanisms of azole resistance in Candida tropicalis isolates causing invasive candidiasis in China. Clin Microbiol Infect. 2019;25(7):885-891. doi:10.1016/j. cmi.2018.11.007

34. Andes DR, Safdar N, Baddley JW, et al. Impact of treatment strategy on outcomes in patients with candidemia and other forms of invasive candidiasis: a patient-level quantitative review of randomized trials. Clin Infect Dis. 2012;54(8):1110-1122. doi:10.1093/cid/cis021

35. Donadu MG, Usai D, Marchetti M, et al. Antifungal activity of oils macerates of North Sardinia plants against Candida species isolated from clinical patients with candidiasis. Nat Prod Res. 2020;34 (22):3280-3284. doi:10.1080/14786419.2018.1557175

36. Donadu MG, Peralta-Ruiz Y, Usai D, et al. Colombian essential oil of ruta graveolens against nosocomial antifungal resistant candida strains. J Fungi (Basel). 2021;7(5):383. doi:10.3390/jof7050383

37. Hamdy R, Fayed B, Hamoda AM, Rawas-Qalaji M, Haider M, Soliman SSM. Essential oil-based design and development of novel anti-candida azoles formulation. Molecules. 2020;25(6):1463. doi:10.3390/molecules25061463

38. Bona E, Cantamessa S, Pavan M, et al. Sensitivity of Candida albicans to essential oils: are they an alternative to antifungal agents? J Appl Microbiol. 2016;121(6):1530-1545. doi:10.1111/ jam. 13282
Infection and Drug Resistance

\section{Publish your work in this journal}

Infection and Drug Resistance is an international, peer-reviewed openaccess journal that focuses on the optimal treatment of infection (bacterial, fungal and viral) and the development and institution of preventive strategies to minimize the development and spread of resistance. The journal is specifically concerned with the epidemiology of antibiotic resistance and the mechanisms of resistance development and diffusion in both hospitals and the community. The manuscript management system is completely online and includes a very quick and fair peerreview system, which is all easy to use. Visit http://www.dovepress.com/ testimonials.php to read real quotes from published authors. 